\title{
Application of inelastic epithermal neutron scattering to the vibrational spectroscopy of adsorbed molecules: Butane physisorbed on graphite (0001) surfaces
}

\author{
R. Wang and $\mathrm{H}$. Taub \\ Department of Physics, University of Missouri-Columbia, Columbia, Missouri 65211 \\ H. J. Lauter \\ Institut Laue-Langevin, 156 X, F-38042 Grenoble Cédex, France \\ J. P. Biberian and J. Suzanne \\ Département de Physique, Case 901, Université d'Aix-Marseille II, 13288 Marseille Cédex 9, France
}

(Received 20 August 1984; accepted 19 December 1984)

\begin{abstract}
Inelastic epithermal neutron scattering (IENS) has been used to investigate the intramolecular vibrations of butane $\left[\mathrm{CH}_{3}\left(\mathrm{CH}_{2}\right)_{2} \mathrm{CH}_{3}\right]$ physisorbed on the (0001) surfaces of a graphite powder. The purpose of these studies was to assess IENS as a vibrational spectroscopy for adsorbed species by using a relatively well-characterized substrate (Carbopack B). The experiments were performed on the IN1 spectrometer located on a beam from the "hot" source at the Institut Laue-Langevin reactor in Grenoble. Film coverages of 1.0 and 3.6 layers were investigated at a temperature of $78 \mathrm{~K}$. The IENS spectra are rich in structure, containing eight vibrational bands in the energy-transfer range from $280-3470 \mathrm{~cm}^{-1}(35-430 \mathrm{meV})$. The similarity in the spectra at the two coverages investigated indicates that in this energy-transfer range the butane intramolecular modes are not strongly perturbed by physisorption on graphite. A detailed comparison is made between the monolayer IENS spectrum and those calculated from models of a free and adsorbed molecule. Both models employ a previously derived intramolecular force field in the normal mode calculation. A third calculation assumes the displacement eigenvectors of the free molecule but replaces the mode eigenfrequencies by their measured Raman and IR values. It reproduces the observed IENS spectra very well. The large number of vibrational bands observed and the close agreement with the calculated spectra suggest butane as a desirable adsorbate for similar experiments on catalytic substrates.
\end{abstract}

\section{INTRODUCTION}

A wide variety of surface spectroscopies are currently being used to probe the dynamics of adsorbed species.' One of the more recent additions to this group has been inelastic epithermal neutron scattering (IENS). ${ }^{2,3}$ The development of spallation neutron sources as well as "hot" sources at steady-state reactors has provided increased fluxes of epithermal neutrons $(E \gtrsim 100 \mathrm{meV})$ necessary for observing excitations in inherently small samples such as adsorbed monolayers.

IENS offers both advantages and disadvantages compared with electron and photon probes as a vibrational spectroscopy for adsorbed species. ${ }^{4}$ Advantages include: (1) the neutron penetrability which permits inelastic spectra to be obtained at virtually any pressure above the adsorbate; (2) the relative ease of calculating vibrational mode cross sections; (3) the absence of dipole and polarizability selection rules; and (4) the sensitivity to the motion of light atoms such as hydrogen.

The penetrability of the neutron is also responsible for the principal disadvantage of IENS as a surface spectroscopy. Lack of surface sensitivity limits applications to substrates of high specific area, typically $\gtrsim 20 \mathrm{~m}^{2} / \mathrm{g}$. While commercial catalysts are certainly among these, they may not be sufficiently well characterized to allow the detailed interpretation of vibrational spectra possible with single-crystal substrates. Another disadvantage of IENS is that the energy resolution $\Delta E / E$ presently attainable is at least an order of magnitude worse than for the electron and optical spectroscopies. ${ }^{5}$

The motivation for the present experiments was to assess the extent to which the advantages of IENS as a surface spectroscopy could be exploited in experiments on a sample as well characterized as possible. Although some IENS spectra had already been obtained from molecules chemisorbed on catalytic substrates, ${ }^{2,3}$ we selected a chemically inert graphitized carbon powder (Carbopack B) as the substrate for our first experiments. Carbopack B provides a large surface area $\left(\sim 80 \mathrm{~m}^{2} / \mathrm{g}\right)$ of highly uniform graphite basal planes (coherence length $\sim 50 \AA) .^{6}$ It was felt that sample homogeneity was of paramount importance initially in order to minimize mode broadening. A greater number of adsorbate modes could then be observed and their relative intensity more reliably compared with calculations. 
For the adsorbate, we selected butane $\left[\mathrm{CH}_{3}-\right.$ $\left(\mathrm{CH}_{2}\right)_{2} \mathrm{CH}_{3}$ ] since both its structure and low-energy vibrational spectrum on graphite had been previously investigated by neutron scattering. ${ }^{4,6-10}$ Elastic diffraction from deuterated butane on the (0001) surfaces of an exfoliated graphite substrate had indicated long-range translational and orientational ordering at submonolayer coverages and temperatures below $116 \mathrm{~K} .{ }^{9,10}$ From profile analysis of the diffraction pattern, it was inferred that the twodimensional unit cell was rectangular centered and contained one molecule lying with its long-axis parallel to the surface.

The experiments presented here were performed with the IN1 spectrometer located on a beam from the hot source of the Institut Laue-Langevin reactor in Grenoble. In the next section, we describe the spectrometer and the sample used in the experiments. The IENS spectra for 1.0 and 3.6 layers of butane on Carbopack B are presented in Sec. III. In Sec. IV, we compare the observed spectra with those calculated from models of a free and adsorbed molecule. Finally, in Sec. V we summarize our results and discuss future prospects of IENS as a vibrational spectroscopy for adsorbed molecules.

\section{EXPERIMENTAL METHODS}

The aluminum sample cell used in these experiments was in the shape of a rectangular slab $70 \mathrm{~mm}$ in height, $48 \mathrm{~mm}$ in width, and $14 \mathrm{~mm}$ thick. The cell had a volume of $33.35 \mathrm{~cm}^{3}$ and contained $13.65 \mathrm{~g}$ of the Carbopack B substrate. From vapor pressure isotherm measurements at $273 \mathrm{~K}$, butane monolayer completion was estimated to occur at an adsorbed volume of $80 \mathrm{~cm}^{3}$ (STP). During the scattering experiments, the sample was maintained at a constant temperature of $78 \mathrm{~K}$.

The IN1 instrument is a triple-axis spectrometer which in our experiments was operated in an energy-loss mode with a copper crystal as monochromator and with a polycrystalline beryllium filter for energy analysis of the scattered neutrons. By changing the reflecting planes of the copper monochromator, various energy ranges of the incident neutrons could be selected as follows:

\begin{tabular}{cccc}
$\begin{array}{c}\text { Reflecting } \\
\text { plane }\end{array}$ & $\Delta E\left(\mathrm{~cm}^{-1}\right)$ & $\Delta E(\mathrm{meV})$ & $Q$ range $\left(\AA^{-1}\right)$ \\
\hline $\mathrm{Cu}(111)$ & 258 to 356 & 32 to 45 & 3.92 to 4.65 \\
$\mathrm{Cu}(002)$ & 339 to 1162 & 42 to 144 & 4.5 to 8.33 \\
$\mathrm{Cu}(220)$ & 887 to 1674 & 110 to 208 & 7.28 to 10.0 \\
$\mathrm{Cu}(331)$ & 1694 to 3630 & 210 to 450 & 10.06 to 14.73
\end{tabular}

A beam monitor was placed between the monochromator and the sample. After suffering an energy-loss collision with the sample, neutrons with a final energy less than $3.8 \mathrm{meV}$ passed through the liquid-nitrogen-cooled beyllium filter before reaching the detector. The detector unit was set at a fixed scattering angle of $35^{\circ}$.

Since the energy resolution of the spectrometer depends on the incident neutron energy as well as the mosaic spread of the monochromator, it differs for each of the monochromator planes used. Almairac et al. ${ }^{11}$ have calculated the energy resolution of the IN1 spectrometer for the four different copper planes which we have used and checked their results in measurements on a powder sample. The instrumental resolution function is described by a Gaussian whose full width at half-maximum (FWHM) increases from 5 to $40 \mathrm{meV}$ (40 to $320 \mathrm{~cm}^{-1}$ ) over the energy transfer range from 40 to $420 \mathrm{meV}$ (320 to 3390 $\mathrm{cm}^{-1}$ ). The ratio of the FWHM of the resolution Gaussian to the energy transfer remains fairly constant at about $9 \%$ over this range.

\section{EXPERIMENTAL RESULTS}

Figure 1 shows the inelastic spectra obtained for 1.0 and 3.6 layers of butane on Carbopack B. In both spectra the inelastic scattering from the bare substrate plotted at the bottom of the figure has been subtracted. Note that as the monochromator reflecting planes are changed, a large difference in the counting rate from the substrate is observed. This results from shifts in the background levels as the counting time for a preset monitor increases at higher energies. Since these shifts occur in all scans, they do not appear in the film spectra after the substrate scattering has been subtracted.

A total of eight vibrational bands are observed in both the 1.0 and 3.6 layer spectra. The similarity between the two spectra suggests that the weak film-substrate

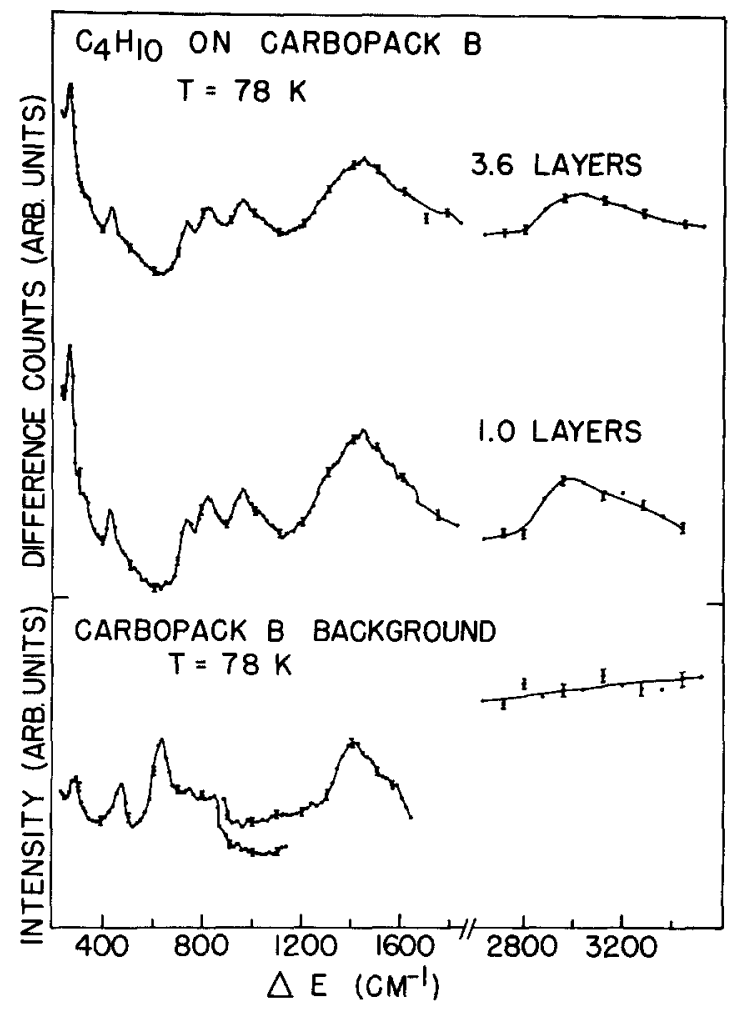

FIG. 1. Inelastic epithermal neutron spectra at coverages of 3.6 and 1.0 layers of butane adsorbed on Carbopack $B$ at $78 \mathrm{~K}$. The background scattering from the substrate which is plotted at the bottom of the figure has been subtracted. 
interaction has little effect on these relatively high-energy intramolecular vibrations of butane. This contrasts with the effect of the substrate at lower energy transfers. In the monolayer spectrum on Carbopack $B$ the lowest intramolecular mode, the $\mathrm{CH}_{2}-\mathrm{CH}_{2}$ torsion, was observed at $157 \mathrm{~cm}^{-1}(19.5 \mathrm{meV})^{6}$ which is perturbed upward by about $20 \%$ from the free-molecule value.

Due to the rather poor energy resolution of the IN1 spectrometer compared to that obtained with optical techniques, identification of the vibrational modes by comparison with Raman and infrared spectra is difficult. More than one mode contributes to each band appearing in the monolayer spectrum above $600 \mathrm{~cm}^{-1}(75 \mathrm{meV})$. In order to identify the contributions to each band, we calculate in the next section all of the intramolecular modes for the simple force constant model of Ref. 7 . Moreover, by combining the calculated mode intensities for inelastic neutron scattering with the observed Raman and infrared mode frequencies, we obtain a neutron spectrum which is in excellent agreement with that observed.

\section{CALCULATION OF SPECTRA}

The method of calculating the vibrational spectrum of the free and adsorbed butane was the same as that used previously. ${ }^{6,7,12}$ The force field of the adsorbed molecule is modeled by including a substrate interaction but neglecting intermolecular couplings which are estimated to be about a factor of 3 smaller. ${ }^{10}$ The molecule is assumed to have the trans configuration and to be in the orientation inferred from elastic diffraction experiments. ${ }^{9,10}$ Its long axis is parallel to the graphite basal plane with the carbon skeletal plane tilted $30^{\circ}$ to the surface. In this orientation, the atoms of the molecule closest to the surface may be considered to belong to one of three different layers. ${ }^{7}$ Each atom within a layer is assumed to be bound by the same force constant to an infinitely massive substrate. The three molecule-substrate force constants were determined from fits to the monolayer butane spectrum at low energy transfers as described in Ref. 7. In the calculation of the free-molecule spectrum, these force constants are set to zero.

The intramolecular force field consisting of eight force constants associated with torsional, bending, and stretching motions of the molecule was taken from the work of Logan et al. ${ }^{13}$ These authors obtained the constants associated with the low-frequency $\mathrm{CH}_{3}$ and $\mathrm{CH}_{2}$ torsional modes and carbon skeletal bending modes from fits to inelastic neutron spectra of solid bulk butane. For the higher-frequency modes, they retained the force constants developed by Snyder and Shachtschneider ${ }^{14}$ from an extensive infrared study.

After solution of the normal mode problem for the adsorbed molecule, the inelastic neutron spectra are calculated in the one-phonon approximation. The incoherent neutron cross section for phonon creation (neutron energyloss scattering) in a lattice with $n$ atoms per unit cell can be expressed as ${ }^{15}$

$$
\begin{aligned}
\frac{d^{2} \sigma^{\mathrm{inc}}}{d \Omega d E}= & \frac{1}{2} \frac{k^{\prime}}{k} \sum_{d=1}^{n} \frac{\sigma_{d}^{\mathrm{inc}}}{M_{d}} e^{-2 W_{d}(\mathbf{Q})} \\
& \times \sum_{j, \mathbf{q}} \frac{\left|\mathbf{Q} \cdot \mathbf{r}_{d}^{j}(\mathbf{q})\right|^{2}}{\omega_{j}(\mathbf{q})}\left[n_{j}(\mathbf{q})+1\right] \delta\left[\omega-\omega_{j}(\mathbf{q})\right],
\end{aligned}
$$

where $\mathbf{k}^{\prime}$ and $\mathbf{k}$ are the final and initial wave vectors, $\mathbf{Q}$ $=\mathbf{k}^{\prime}-\mathbf{k}$ is the momentum transfer, and $q$ is the phonon wave vector. The eigenvector and eigenfrequency of atom $d$ in the $j$ th normal mode are $\mathbf{r}_{d}^{j}(\mathbf{q})$ and $\omega_{j}(\mathbf{q})$, respectively. The $d$ th atom has mass $M_{d}$, incoherent cross section $\sigma_{d}^{\text {inc }}$, and Debye-Waller factor $W_{d}$. The phonon occupation number $n_{j}(\mathbf{q})$ is $\left\{\exp \left(h \omega_{j}(\mathbf{q}) / k_{B} T\right]-1\right\}^{-1}$.

Equation (1) greatly simplifies for the model of the adsorbed molecule which we are considering here. First, since each molecule is assumed to vibrate independently, the eigenvectors $\mathbf{r}_{d}^{j}$ and eigenfrequencies $\omega_{j}$ are $q$ independent. Second, due to the large incoherent cross section of hydrogen and its light mass, the contribution of the butane carbon atoms to the cross section can be neglected with an error of less than $1 \% .^{16}$ Third, because the substrate is assumed to be isotropic, the term $\left|\mathbf{Q} \cdot \mathbf{r}_{d}^{j}\right|^{2}$ in Eq. (1) can be replaced by its average over a sphere $\frac{1}{3} Q^{2}\left(r_{d}^{j}\right)^{2}$. With these approximations, the intensity of the $j$ th mode is

$$
I_{j} \propto \frac{k^{\prime}}{k} \frac{Q^{2}\left[n\left(\omega_{j}\right)+1\right]}{\omega_{j}} \sum_{d}\left(r_{d}^{j}\right)^{2} .
$$

The spectrum calculated from Eq. (2) was folded with the instrumental resolution function described in Sec. II. In addition, a correction was made for the energy dependence of the beam monitor efficiency which varies as $1 /$ $k$ where $k$ is the incident wave vector. For comparison with the observed spectrum, the calculated spectra are scaled up by a factor $k$.

The spectra calculated for the free and adsorbed molecules are shown in the middle portion of Fig. 2. For the free butane molecule consisting of 14 atoms, there are 36 intramolecular vibrational modes. All but the two lowest torsional modes of the $\mathrm{CH}_{2}$ and $\mathrm{CH}_{3}$ groups lie in the energy transfer range of Fig. 2. As a result of folding with the instrumental resolution function, these modes appear in ten bands labeled $A^{\prime}$ through $K^{\prime}$.

As anticipated, there is little difference between the spectra calculated for the free and adsorbed molecule. The latter exhibits a small upward shift in the bands labeled $A^{\prime}$ and $D^{\prime}$ corresponding to the upper $\mathrm{CH}_{3}$ torsion and the $\mathrm{CH}_{2}$ rocking mode, respectively. The small perturbation calculated for these modes is consistent with the lack of coverage dependence in the observed spectra. If the film-substrate bonds significantly perturbed the intramolecular modes, one would expect differences between the monolayer and 3.6-layer spectra since the thicker film contains a smaller fraction of these bonds.

The calculated spectra agree fairly well with the observed spectrum for 1.0 layer of butane on Carbopack B which appears again at the top of Fig. 2 to facilitate comparison. Based on their order of appearance, we can make a preliminary identification of the bands as shown 


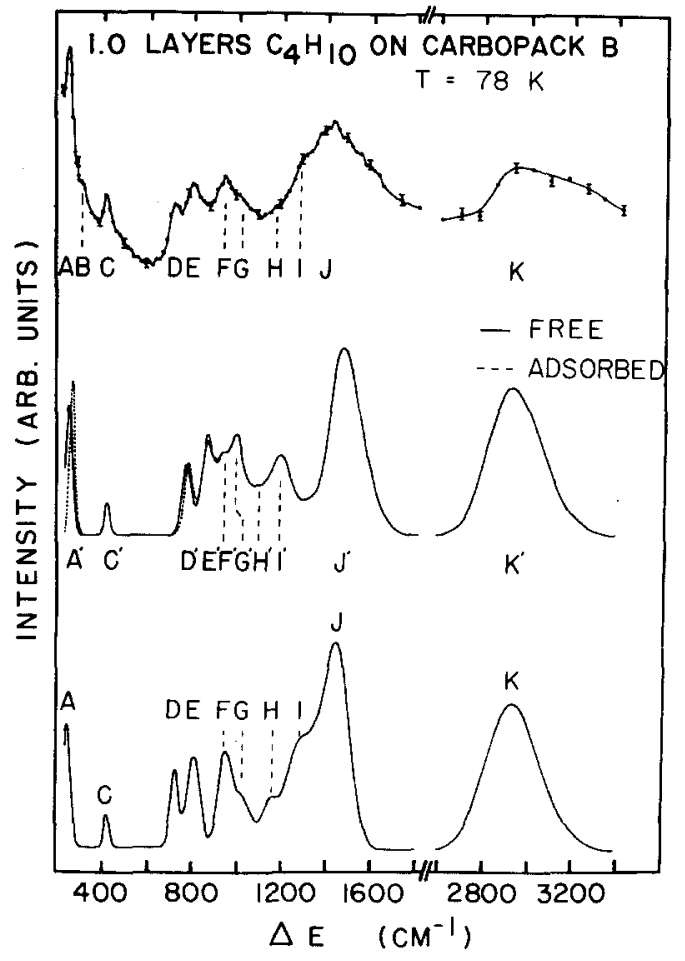

FIG. 2. Comparison of observed and calculated inelastic epithermal neutron spectra for monolayer butane. The observed spectrum (upper portion) is replotted from Fig. 1. In the middle portion of the figure, the dashed line labeled ADSORBED is the calculated spectrum based on the model used to fit the low-energy monolayer butane spectrum in Ref. 7. The spectrum drawn with the solid line labeled FREE neglects the film-substrate interaction. The calculated spectrum at the bottom of the figure uses the optically measured band frequencies and the calculated eigenvectors of the modes as described in the text. All calculated spectra have been folded with the instrumental resolution function and corrected for monitor efficiency. Due to the close resemblance between the observed spectrum (top) and the calculated hybrid spectrum (bottom), corresponding bands have been labeled with the same letter (unprimed). Primed letters label corresponding bands in the other calculated spectra (middle) where the agreement with the observed spectrum is somewhat worse.

( $A$ with $A^{\prime}, C$ with $C^{\prime}$, etc.). Although the observed band $B$ occurs near the frequency calculated for the carbon bending mode, its intensity is too weak to appear in the calculated spectra.

The largest discrepancy with the monolayer spectrum occurs near $1200 \mathrm{~cm}^{-1}$ where a single broad band is observed instead of the two calculated bands $\left(I^{\prime}\right.$ and $\left.J^{\prime}\right)$. We believe this results from defects in the assumed intramolecular force field. In the low-energy portion of the 1.0-layer butane spectrum, the $\mathrm{CH}_{3}$ torsion $(A)$ and carbon bending modes ( $B$ and $C$ ) appear to be riding on a monotonically decreasing background which is absent in the calculated spectra. Since this background increases somewhat at 3.6 layers (see Fig. 1), we tentatively attribute it to multiphonon processes not included in the onephonon cross section of Eq. (2).

Rather than refine the intramolecular force field to improve the agreement with experiment, we have compared the mode frequencies observed in the monolayer neutron spectrum with those previously found in higherresolution Raman and infrared measurements. This was done by calculating a hybrid spectrum in which the mode eigenfrequencies are taken to be the optically measured values while the corresponding eigenvectors are those from the normal mode calculation of the free molecule. Previous mode assignments ${ }^{14,17,18}$ were used to match the observed eigenfrequencies to the calculated eigenvectors for the 36 intramolecular butane modes. ${ }^{19}$

The hybrid spectrum is shown at the bottom of Fig. 2 after folding with the instrumental resolution function. The agreement with the monolayer butane neutron spectrum at the top of the figure is better than for the calculated spectra of the free and adsorbed molecule. The band positions inferred from the individual Raman and IR mode frequencies match those in the neutron spectrum to within experimental error. This supports our earlier contention that the poor agreement between the frequencies of the $I$ and $I^{\prime}$ bands resulted from the intramolecular force constants used in the calculated spectra. There is even evidence in the hybrid spectrum of some of the more subtle features observed such as the shoulders labeled $G, H$, and $I$.

However, some features of the monolayer spectrum are not reproduced. The large dip in intensity predicted between bands $E$ and $F$ is not observed. Also band $J$ appears to have a high-energy tail which is not present in the hybrid spectrum. The source of this apparent mode broadening is not understood at the present time. Another discrepancy with the hybrid spectrum is the asymmetric shape observed for band $K$ corresponding to the $\mathrm{C}-\mathrm{H}$ stretching modes. This may result from second harmonics of the intense band $J$ of bending modes.

\section{v. SUMMARY}

These experiments demonstrate that presently available epithermal neutron sources provide sufficient flux to observe nearly all of the vibrational states of butane adsorbed on a high-surface-area graphite powder. Even the high-frequency $\mathrm{C}-\mathrm{H}$ stretching modes involving relatively small amplitude hydrogen motion can be seen. The weak binding of butane to the graphite basal plane surfaces leaves the intramolecular vibrations essentially unperturbed. This has enabled us to make a detailed comparison of the observed monolayer spectrum with those calculated from a model of the intramolecular force field and with a hybrid spectrum using mode frequencies measured by optical techniques. The results indicate that very good agreement is possible between the observed and calculated relative mode intensities for a well-characterized system. Finally, the large number of vibrational bands and the close agreement with the calculated spectra suggest butane as a desirable adsorbate for similar experiments on catalytic substrates. The IENS spectra of physisorbed butane presented here will provide a useful standard for comparison with butane spectra on catalytic substrates.

\section{ACKNOWLEDGMENTS}

We are indebted to $\mathrm{H}$. Kim for suggesting the calculation of the hybrid spectrum and for providing the computer code used in the normal mode calculations. 
This work was supported in part by National Science Foundation Grants DMR-7905958, INT-8012228, and DMR- 8304366 and by a grant of the Petroleum Research Fund, administered by the American Chemical Society.

${ }^{1}$ For a review of various surface spectroscopies including neutron scattering see Vibrational Spectroscopies for Adsorbed Species, ACS Symposium Series, No. 137, edited by A. T. Bell and M. L. Hair (American Chemical Society, Washington, D.C., 1980).

${ }^{2}$ H. Jobic, J. Tomkinson, J. P. Candy, P. Fouilloux, and A. J. Renouprez, Surf. Sci. 95, 496 (1980).

${ }^{3}$ H. Jobic and A. Renouprez, Surf. Sci. 111, 53 (1981).

${ }^{4}$ H. Taub, in Ref. 1.

${ }^{5}$ Improvements in epithermal neutron sources and spectrometers are occurring rapidly. For example, a recently completed upgrade of the three-axis IN1 spectrometer at the Institut Laue-Langevin in Grenoble should result in significantly better resolution and scattered intensities.

${ }^{6}$ H. Taub, H. R. Danner, Y. P. Sharma, H. L. McMurry, and R. M. Brugger, Phys. Rev. Lett. 39, 215 (1977); Surf. Sci. 76, 50 (1978).

${ }^{7}$ R. Wang, H. R. Danner, and H. Taub, in Ordering in Two Dimensions, edited by S. K. Sinha (North-Holland, New York, 1980), p. 219.

${ }^{8}$ H. Kim and H. R. Danner, J. Chem. Phys. 77, 4169 (182).

${ }^{9}$ G. J. Trott, H. Taub, F. Y, Hansen, and H. R. Danner, Chem. Phys. Lett. 78, 504 (1981).
${ }^{10}$ G. J. Trott, Ph.D. thesis, University of Missouri-Columbia, 1981.

"R. Almairac, J. L. Prefaut, M. Galtier, C. Benoit, A. Montaner, and H. J. Lauter, Mol. Cryst. 69, 177 (1981).

${ }^{12} \mathrm{H}$. Kim, Biopolymers 21, 2083 (1982).

${ }^{13}$ K. W. Logan, H. R. Danner, J. D. Gault, and H. Kim, J. Chem. Phys. 59, 2305 (1973). The only change which we have made in their force constant model is in the torsional constant involving the interior carbon atoms. It has been adjusted to fit the $\mathrm{CH}_{2}-\mathrm{CH}_{2}$ torsion observed in the bulk liquid at $130 \mathrm{~cm}^{-1}$ as reported in $\mathrm{K}$. A. Strong, AEC Report IN-1237, Idaho Nuclear Corp.

${ }^{14}$ R. G. Synder and J. H. Schachtschneider, Spectrochim. Acta 19, 85 (1963).

${ }^{15}$ W. Marshall and S. W. Lovesey, Theory of Thermal Neutron Scattering (Oxford University, Oxford, 1971), p. 89.

${ }^{16}$ B. Hudson, A. Warshel, and R. G. Gordon, J. Chem. Phys. 61, 2929 (1974).

${ }^{17}$ R. G. Synder, J. Chem. Phys. 47, 1316 (1967).

${ }^{18}$ T. Shimanouchi, H. Matsuura, Y. Ogawa, and I. Harada, J. Phys. Chem. Ref. Data 7, 1323 (1978).

${ }^{19}$ A table comparing the mode frequencies calculated for a free butane molecule with the optically measured values is given in $\mathbf{R}$. Wang, Ph.D. thesis, University of Missouri-Columbia, 1983. This reference also contains a description of the mode eigenvectors, their symmetry, and atomic displacement patterns for selected modes. 